\title{
Getting a clearer picture
}

\section{Adaptive optics has a huge range of applications. Nadya Anscombe talks to Robert Tyson, associate professor at the University of North Carolina at Charlotte in the USA, to find out his views on its future.}

\section{What are the applications of adaptive optics today?}

The technology behind adaptive optics was originally developed by the defence industry for propagating laser beams into space, but has since become an essential technology in astronomy. Every telescope larger than $4 \mathrm{~m}$ in diameter needs adaptive optics to correct for the 'twinkling' of stars due to atmospheric distortions. Throughout the 1980s, engineers in the field of astronomy worked to solve the problem of not having an object bright enough to use as a reference. The development of laser guide stars, in which an artificial beacon of scattered light is placed high in the atmosphere, opened the door for the high-resolution imaging of very dim astronomical objects. A new exciting application for adaptive optics is in ophthalmology, where it can be used to reduce optical aberrations and therefore give sharper pictures of the retina. The ultimate aim is to have a camera in every ophthalmologist's office, but the technology is still under development. Free-space laser communications is another developing application for adaptive optics. Here the technology is used to compensate for atmospheric interference such as turbulence, as well as potentially correcting for aberrations caused by fog, rain and dust particles. Adaptive optics is also used in manufacturing to change the focus of a laser beam without changing lenses, in optical coherence tomography to obtain sharper images, and also in scanning microscope technology. Perhaps the most challenging application for adaptive optics at the moment is in the Airborne Laser Testbed, which last year successfully shot down a missile in flight for the first time. This application features an incredibly complex adaptive optics system working in the harshest of environments.

\section{Apart from in astronomy, most} applications of adaptive optics are still in development. Why have they not yet been commercially successful?

Each market has its own requirements. In the ophthalmology market, developers need to stabilize the electronics and put the current laboratory systems into a

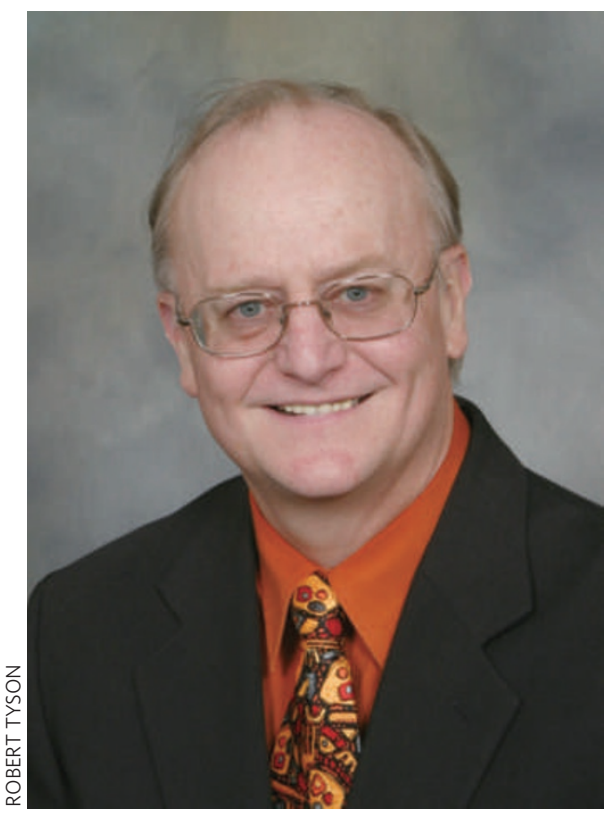

Robert Tyson: "The challenge now is to build control schemes than can handle the vast amounts of data produced by adaptive optics systems."

compact, sterile box that can be used by the ophthalmologist without the need for a technician. Costs also need to come down, but this will happen once massproduction begins. Despite ophthalmology being a highly specialized field, adaptive optics can make a huge difference, enabling ophthalmologists to see the retina more clearly and make earlier diagnoses. In free-space communication, the technology is available but the market does not yet exist. Most applications will make do with a wireless connection, with very few currently requiring the extra bandwidth that laser communication can provide. In this industry, it is a case of "if they come, we will build it".

What are the main technological hurdles that need to be overcome? When adaptive optics was first realized, making and controlling the deformable mirrors were the main challenges. In the late 1970 s to 1980 s, the bottleneck was the wavefront sensor technology. This is no longer the case, thanks to the availability of mature deformable mirror technology and the plethora of phenomenal sensors and cameras on the market. The challenge now is to build control schemes than can handle the vast amounts of data produced by adaptive optics systems. The technology has become so complex that simply scaling up the old algorithms used in previous systems is no longer an option. Hierarchical controllers and algorithms are needed to work out which aberrations are important and which to leave out. Although adaptive optics technology was first envisioned in the 1950 s, it only became practical with advances in computing power. Now it is computing power that seems to be the bottleneck, but the area is moving quickly and progress is constantly being made. For example, the adaptive optics system for the European Extremely Large Telescope (diameter of $42 \mathrm{~m}$ ) is currently under development. It will use multiple guide stars of different wavelengths as well as a variety of large and small deformable mirrors. The computing requirements for this project are enormous, but progress is being made.

\section{What new applications do you} envisage for adaptive optics?

Current interest in adaptive optics is at an unprecedented level. The number of research papers published has increased exponentially in recent years, and the possibilities in this field really are endless. I am seeing a cross-fertilization of ideas, whereby researchers from different disciplines are coming together and realizing what adaptive optics could do for them. In defence, the military is interested in using adaptive optics in communications systems at the front line. Adaptive optics could even go back to its roots and be used as a directed energy source to shoot down space debris from a ground-based system, or it could be used to make super-resolution spectacles, giving the user 20:20 vision at the touch of a button. There are countless applications that have yet to be discovered.

\section{NADYA ANSCOMBE}

Nadya Anscombe is a freelance journalist based in the United Kingdom. 